\title{
NOTAS SOBRE UN GRUPO DE BALLESTAS ESPAÑOLAS PARA EL EMPERADOR MAXIMILIANO I DE AUSTRIA
}

\author{
POR \\ ALVARO SOLER DEL CAMPO
}

\section{RESUMEN - ABSTRACT}

Se discute un grupo de ballestas pertenecientes a Maximiliano I de Austria. La aparición de marcas no documentadas hasta el momento confirman que todos sus elementos fueron construídos en España, resaltando, igualmente, el papel del reino de Aragón como centro productor.

The author describes a set of crossbows belonging to Maximilian I of Austria. Trademarks hitherto unobserved confirm that they were manufactured in Spain, specially in Aragon.

\section{PALABRAS CLAVE - KEY WORDS}

Ballestería. Centros de producción. Marcas. Reino de Aragón. Maximiliano I de Austria. Hofjagd und Rüstkammer. Viena.

Crossbow manufacture. Production centres. Trademarks. Reign of Aragon. Maximiliano I of Austria. Hofjagd und Rüstkammer, Viena.

Entre las armas pertenecientes al emperador Maximiliano I de Austria (1459-1493-1519) conservadas en la Hofjagd und Rüstkammer del Kunsthistorisches Museum de Viena, la antigua armería imperial, se encuentra un grupo de ballestas españolas, datadas hacia 1508-1515, compuesto por los números de catálogo D.3, D.16, D.17 y D.181. Se trata de cuatro ballestas tipológicamente análogas, diferenciadas por pequeños rasgos formales, no analizados aquí en detalle, referentes a los estribos, llaves y secciones transversales de las cureñas o tableros, pero cuya decoración e inscripciones señalan inequívocamente como propietario a Maximiliano I.

Todas ellas siguen la tipología de las ballestas españolas del momento, aunque ésta ya aparece esbozada en sus rasgos principales en la miniatura hispana del siglo XIII. La sección longitudinal de las cureñas se caracteriza por la rectitud del perfil superior y la sinuosidad del perfil inferior. Este último muestra una curva ascendente desde el extremo anterior para dar paso a la caja de la nuez y facilitar al mismo tiempo que el arma pueda ser empuñada con la mano izquierda. Desde la caja de la nuez pueden responder a dos variantes. Según la primera, el perfil inferior adopta de nuevo una curva ascendente entre los dos extremos de la rabera, o mitad inferior del tablero. En la segunda, el perfil inferior responde a una línea progesivamente ascendente desde la caja de la nuez hasta el extremo inferior de la rabera.

\footnotetext{
1 Thomas y Gamber, 1976: 205-206.
} 


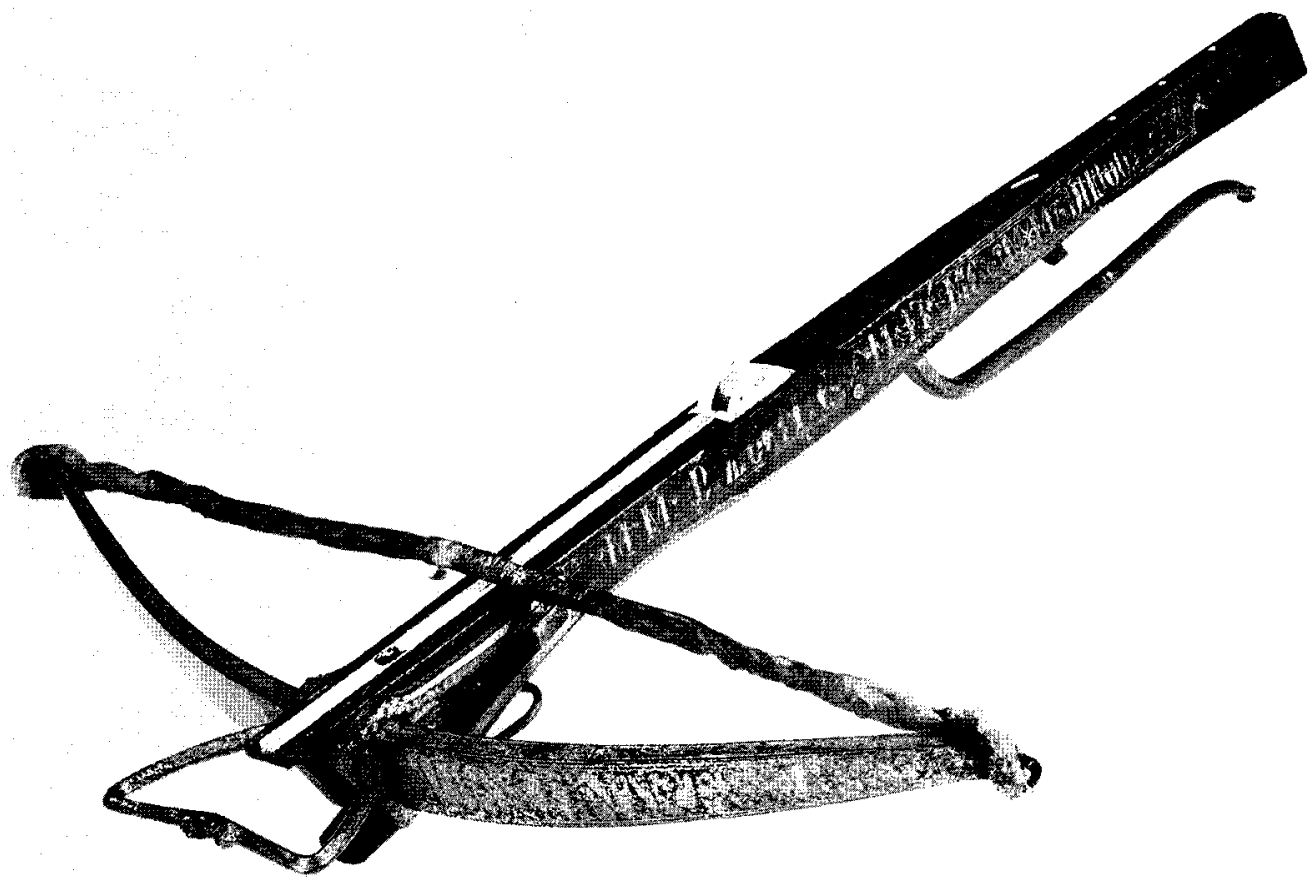

Fig. 1. Ballesta de Maximiliano I. Kunsthistorisches Museum. Hofjagd und Rüstkammer. Cat. D.3.

Salvo en el caso de la ballesta D.17, la decoración toma como base la policromía de las cureñas, en las que destacan la pintura roja que actúa como fondo, y las supericies doradas y/o grabadas de la verga, estribo, flores y llave. Sobre el fondo rojo de las cureñas se disponen inscripciones que alternan letras doradas y blancas limitadas por cartelas festoneadas en blanco, color utilizado igualmente para las lacerías y motivos florales que se intercalan entre algunas piezas. Como complemento de la decoración pintada también se debe señalar el color blancuzco de las guarniciones de hueso, bien en elementos funcionales, como el canal para soportar la saeta, o meramente decorativos, como el filete embutido que recorre el eje de la rabera.

El interés de estas ballestas radica en las inscripciones que señalan a Maximiliano I como su propietario y en las marcas situadas en los arcos, o, en un caso, en la rabera del tablero, que indican que se trata de una producción de origen español. Hasta ahora se había considerado que sólo los arcos eran de segura procedencia española, mientras que las cureñas se atribuían a un maestro español y la decoración habían sido ejecutada en el Tirol. El arco de la ballesta D.3 muestra grabadas las incripciones: «SI DEVS PRO NOBIS - QVIS CONTRA NOS (exterior) / DILIGENTES MM - MM ME DILIGO (interior)». Estas se repiten básicamente en la cureña: «DILIGENTES ME DILIGO (cara superior) / MM - MM DILIGENTES MM ME MM DILIGO (lateral izquierdo) / MM DILIGENTES MM ME MM DILIGO - MM (lateral derecho)». El arco de D.16 introduce una invocación al texto ya señalado: «SI DEVS PRO NOBIS QVIS CONTRA NOS (exterior, pero invertida respecto a la cara interior) / SI DEVS PRO NOBIS QVIS CONTRA NOS (interior) / IHESVS MARIA (borde superior)»².

2 Por un error tipográfico, en el catálogo de la Hofjagd und Rüstkammer se han cambiado las inscripciones de D.16 por D.18 y viceversa. Ver: Thomas y Gamber, 1976: 206. 


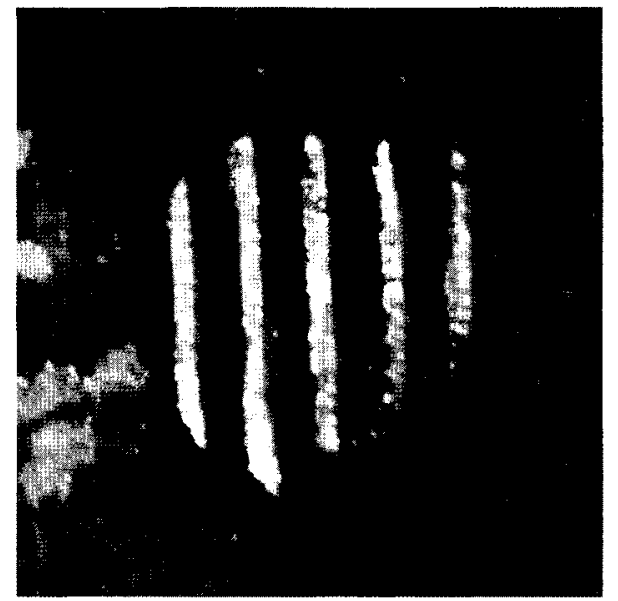

Fig. 2. Ballesta. K.H.M., cat. D.3. Marca con el escudo de Aragón sobre la cureña. Detalle ampliado.

Las inscripciones y la decoración de D.17 difieren del resto del grupo por la utilización del escudo y del mote imperial «HALT MAS», adoptado por Maximiliano I desde 1509. Carece por tanto del carácter religioso y profiláctico de las inscripciones anteriores. También se diferencia porque el arco no está grabado y dorado, si no pintado conjugando águilas bicéfalas y el mote HALT MAS en el exterior y en el interior de la verga, a derecha e izquierda. La cureña tampoco está pintada en rojo, sino en su color, con guarniciones metálicas pavonadas, repitiendo el mote y el emblema imperial. Es posible que con ello se quisiera dar un especial valor a este arma en concreto, o que formara parte de una otra serie parcialmente perdida.

La ballesta D.18 combina en parte las inscripciones de las anteriores. En el arco la inscripción de la cara externa está invertida en la posición de uso, pero no la interna: «HALT MAES - HALT MAES (cara externa) / SI DEVS PRO NOBIS - QVIS CONTRA NOS (cara interna)». La decoración del arco introduce además chispas del Toisón de Oro enmarcando las inscripciones. En la cureña se repite básicamente el mismo texto: «SI DEVS PRO MOBIS QVIS CONTRA NOS (lado izquierdo) / DA PACEM DOMINE IN DIEB(VS) NOSTRIS (lado derecho) / HALT MAS (cara superior).

Relacionadas con el grupo de Viena deben citarse otras tres ballestas. La primera se conserva en el Musée de l'Armée de París (cat. L.43), habiendo sido reconocida como parte del mismo grupo por tener la inscripción «SI DEVS PRO NOBIS QUIS CONTRA NOS» en el interior del $\operatorname{arco}^{3}$. La segunda y la tercera se conservan en el Deutsches Historisches Museum de Berlín, Zeughaussammlung, procedentes de la actual Hofjagd und Rüstkammer vienesa. Fueron llevadas a París desde Viena o Ambras bajo Napoleón I, pasando posteriormente a Berlín en $1815^{4}$. La segunda (D.H.M., cat. W 82.1, ex PC.8556) repite de nuevo la misma inscripción en la cara interior del arco: «SI DEVS PRO NOBIS QVIS CONT(RA) NOS». La caja de esta última también está pintada en rojo y marrón siguiendo a los ejemplares vieneses, mostrando, igualmente, una inscripción parcialmente legible: «QVID-QVID AGIS....TER / AGAS-ETRE SPICE FIEM» ${ }^{5}$. En la tercera (D.H.M., Cat. W.835 ex. PC.8555) se aprecian también restos de decoración pintada en la caja, pero difiere por la inscripción: «SPERO LUCEM / MATER DEI MEMENTO MEI».

3 Thomas y Gamber, 1976: 206.

4 Müller, 1994: 62, lám.75; 151; 232; 277 nota 332.

5 Hilt, $1876: 111$, nr.717. 


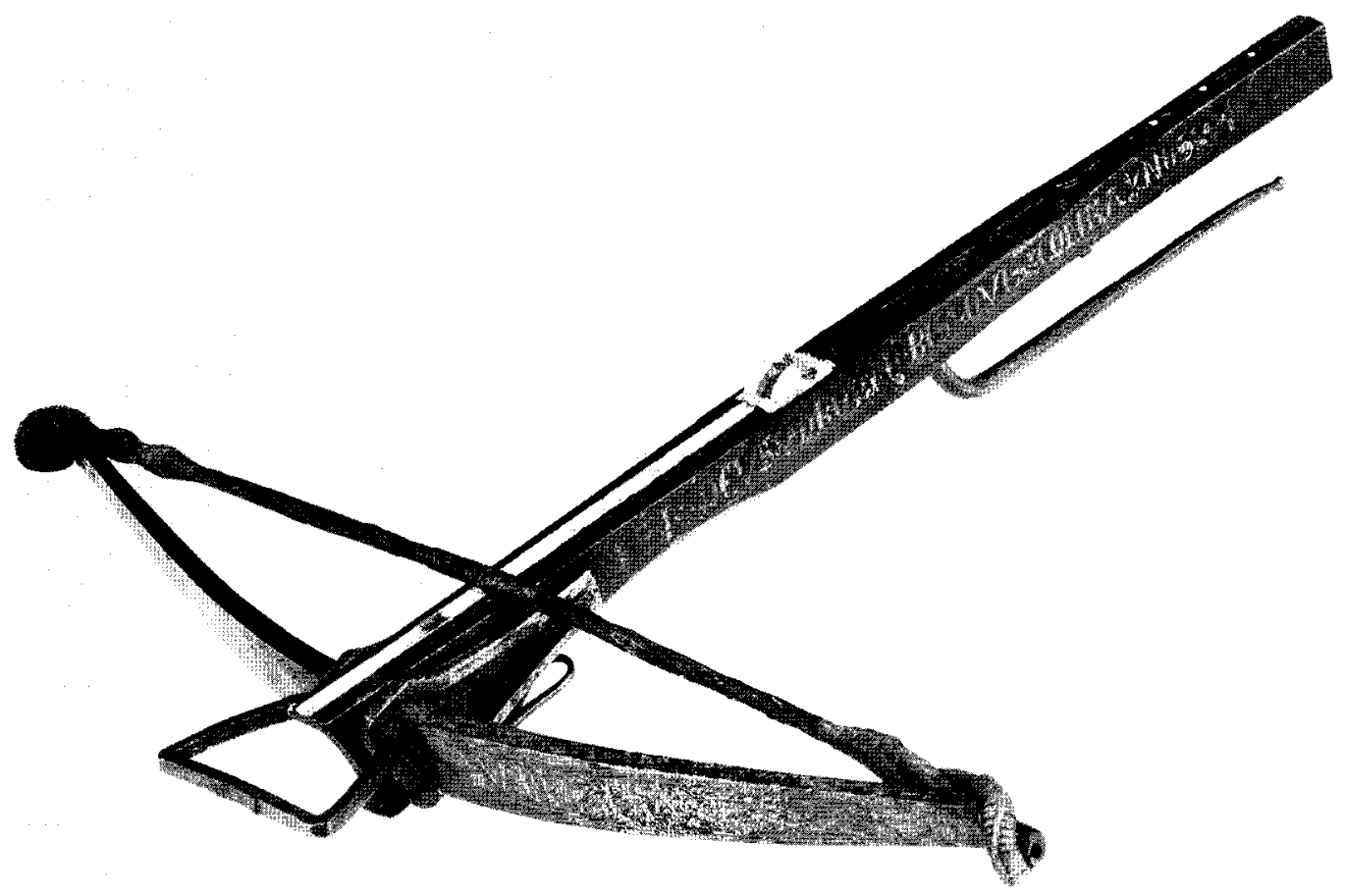

Fig. 3. Ballesta de Maximiliano I. Kunsthistorisches Museum. Hofjagd und Rüstkammer. Cat. D.18.

Estas siete ballestas no sólo tienen en común las inscripciones relativas a Maximiliano I. Todas ellas ostentan la misma marca repetida dos veces sobre cada uno de los arcos: un escudete heráldico, con cuatro barras verticales cubriendo el campo. Este punzón se corresponde con el escudo del Reino de Aragón, documentado desde un sello de Ramón Berenguer IV (1131-1162). Desde 1343, durante el reinado de Pedro el Ceremonioso, fue adoptado definitivamente como escudo real, pasando a la casa de Trastámara y a la Corona de España ${ }^{6}$. Por ello no se ha dudado de la procedencia española de sus arcos.

Las cureñas se han atribuído por razones estilísticas a un taller español, mientras que su decoración se ha considerado, sin embargo, de procedencia tirolesa, posiblemente hechas en Innsbruck dentro de la corte 7 . Un nuevo análisis del conjunto vienés revela la presencia de marcas no recogidas con anterioridad que confirman la cuestión de su autoría y procedencia. La más significativa es la repetición en la ballesta D.18 de la marca de Aragón, concretamente en la cara inferior del extremo posterior de la rabera.

La presencia de una marca en el tablero de una ballesta es una práctica muy poco usual, documentada en la ballesta D.18, pero no en las seis restantes. Entre ellas si se han documentado dos marcas no recogidas hasta el momento, que indican la actividad de al menos dos ballesteros distintos dentro de este conjunto. Debemos presumir que ambas corresponden también a talleres aragoneses. 


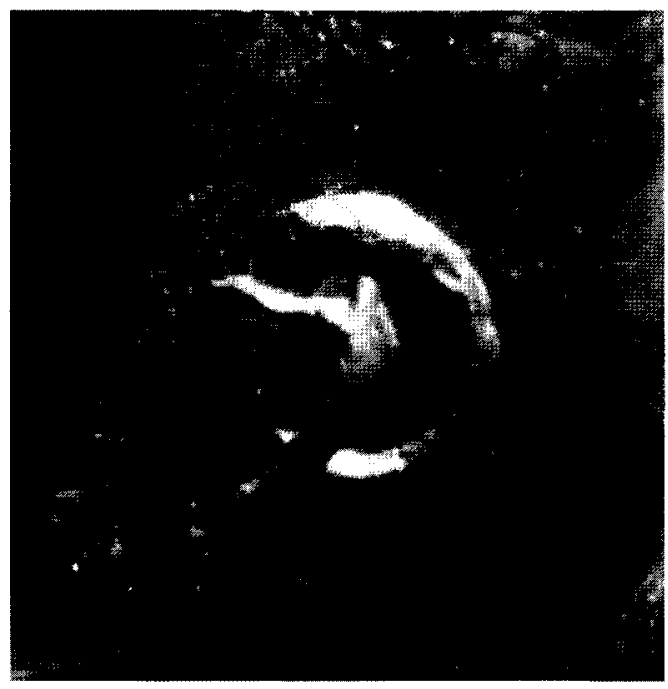

Fig.4. Ballesta. K.H.M. D.18. Marca sobre la llave. Detalle ampliado.

La primera se trata de una marca en forma de media luna, situada en las llaves de las ballestas D. 3 de Viena y L.43 de París. La segunda responde a un cuerpo de apariencia circular dotado de radiantes en la parte superior, presente en la ballesta berlinesa W.835. La combinación de este último punzón con la marca a manera de el escudo de Aragón se encuentra también en a ballesta J.17 de la Real Armería de Madrid, procedente de la armería imperial de Carlos V pero sin conexión con el grupo tratado ${ }^{8}$. La Real Armería conserva, junto con la ya señalada, cinco ballestas tipológicamente análogas (cat. J.8, J.10, J.12, J.16, J.17, J.22), pero sólo la ballesta J.8 vuelve a mostrar una marca con radiantes similar a las ballestas W.835 de Berlín y J.17 de Madrid, pero en esta ocasión carece del escudo de Aragón.

La repetición del escudo de Aragón en el arco y en el tablero de la ballesta D.18, junto con ciertos rasgos e incorreciones de la decoración pintada, aportan una nueva luz sobre las producciones de armas de lujo, su comisión y ejecución. Igualmente son importantes para continuar delimitando el papel del reino aragonés como centro productor y exportador de armas de calidad.

En primer lugar, la marca en el tablero de D.18 señala inequívocamente que la totalidad del arma fue hecha o ensamblada en España por un taller aragonés. Las marcas restantes deben ser consideradas por tanto españolas, indicando así mismo el concurso de diferentes ballesteros o maestros especializados en la elaboración de los distintos elementos.

La unicidad constructiva de estas ballestas también parece ser corroborada por la decoración pintada. En la ballesta D.3 se aprecia claramente cómo la pintura de la cureña se superpone a las guarniciones metálicas de la caja de la nuez. La decoración pintada se adapta por tanto al soporte, es decir, no se han concebido paralelamente forma y decoración, ni se han llegado a desmontar los elementos metálicos del arma para ejecutar con mayor precisión la decoración, o utilizar ésta para diferenciar los tipos de soporte, prácticas usuales en la elaboración de armas de lujo.

Estos rasgos y el carácter tirolés de la decoración, reconocido por cuestiones estilísticas, sugieren que estas ballestas o bien fueron enviadas terminadas y montadas desde España, o

\footnotetext{
8 Valencia de Don Juan, I989: 281.
} 
desmontadas para ser ensambladas y posteriormente decoradas en el Tirol. El hecho más significativo es que hipotéticamente pudieron haber sido encargadas y enviadas sin decorar, es decir, como productos en bruto que todavía debían ser terminados antes de su uso por Maximiliano I. Ello supone un matiz importante sobre el concepto de producción en un arma de lujo, generalmente caracterizados por una unicidad en la elaboración. Esta solía realizarse en talleres en los que podían intervenir varios especialistas en los diversos componentes o técnicas decorativas, pero generalmente dentro de un mismo centro productor. En este caso nos encontramos ante una nueva decoración, ante la constatación material de la elaboración de un arma en dos fases, cada una de ellas realizada en centros muy distantes entre si.

La ballesta D. 18 también abre otros interrogantes por sus inscripciones grabadas y pintadas. Las grafías incorrectas MOBIS y MAES, sugieren la posible intervención de artistas que no conocen bien ni el latín ni el alemán. El problema se subsana en las ballestas restantes, por lo que puede ser interpretado como un doble error no repetido, o como la intervención de dos manos distintas: un grabador que conoce el latín pero no el alemán, y un pintor siguiendo la tradición tirolesa que conoce bien el alemán pero no el latín.

Independientemente de ello, cabe anotar que la presencia de lemas o del nombre del propietario en las ballestas, tampoco habrían sido ajenas a la tradición aragonesa según indica la documentación desde mediados del siglo XIV ${ }^{9}$.

El uso como marca de las armas de Aragón permite continuar perfilando la importancia del reino como centro productor de gran calidad durante el último tercio del siglo XV e inicios del XVI, con una producción característica no bien conocida necesitada de estudio y eclipsada por otros centros continentales de mayor importancia. Hacia 1490-1510 la frontera de la Corona de Aragón se trazaba entre las localidades actuales de Sos del Rey Católico, Calatayud, Teruel y Orihuela, abarcando, por tanto, Valencia, Barcelona y las Islas Baleares. La actividad de algunos de las principales ciudades como centros de producción o exportación de armas es conocida documentalmente desde la Edad Media, como en los casos de Barcelona o Valencia ${ }^{10}$. La documentación medieval indica que la producción de ballestas dentro del reino durante la Baja Edad Media se realizaba fundamentalmente en Valencia y en las Islas Baleares. Valencia fue un importante centro exportador de armas documentado en fecha tan temprana como 1394, época en que las armas constituyen la principal mercancía exportada hacia Flandes. Las ballestas, espadas, y piezas de armaduras eran las más importantes, sobre todo con motivo de la Guerra de los Cien Años (1328-1453).

Mallorca también actuó desde el siglo XIV como un importante centro exportador de armas en el Mediterráneo occidental. Entre los años 1374-1419 destacaba por exportar componentes de ballestas generalmente enviados a Barcelona y Valencia, donde se terminaba de construir el arma. Este dato es importante porque corrobora documentalmente la producción de ballestas en dos centros y en dos fases desde fecha temprana, al igual que sucedió posteriormente en el encargo para Maximiliano I. Valgan como ejemplos registrados los envíos, citados por Alomar, de 15.473 cureñas a Barcelona, 9.180 a Valencia, 2.000 a Flandes y 500 a Castilla. Según estas cifras la mayor parte de las exportaciones mallorquinas tenían Barcelona como cabeza, predominando en el comercio de armas las cureñas de ballestas ${ }^{11}$.

Carecemos de elementos de juicio para poder afirmar que las ballestas encargadas para Maximiliano procedían de Valencia o Mallorca. Ambas eran conocidas como centros productores de estas armas, con experiencia en la exportación de ballestas hacia Europa, fundamentalmente a Flandes. El paralelo con las armas encargadas para Maximiliano I es evidente y muy sugerente, permitiendo pensar, en la actualidad sólo como hipótesis, en un posible

9 Alomar, 1995: 132, nota 294.

10 Hinojosa Montalvo, 1972. Hinojosa Montalvo, 1975: vol.I, 125-137.

11 Para la cuestión de las exportaciones en la Edad Media ver: Alomar, 1995: 117-118; Sevillano Colom, 1971:431-520; Hinojosa Montalvo, 1975: vol.I, 125-137. 
origen valenciano o mallorquín para todos los elementos de estas ballestas decoradas a posteriori según la moda del Tirol.

\section{AGRADECIMIENTOS}

Quiero agradecer a la Subdirección General de Información e Investigación del Ministerio de Educación y Cultura la concesión de una beca para el estudio de los fondos españoles de la Hofjagd und Rüstkammer del Kunsthistorisches Museum de Viena. Agradezco también su ayuda y apreciaciones a los doctores Christian Beaufort-Spontin y Matthias Pfaffenbichler de la armería vienesa, a Gerhard Quaas del Deutsches Historisches Museum de Berlín y a Jean-Pierre Reversau del Musée de l’Armée de París.

\section{Alvaro SOlER DEL CAMPo}

Conservador de la Real Armería. Patrimonio Nacional. Palacio Real.

C/Bailén s/n-28071 Madrid - e-mail:asoler.armeria@patrimonionacional.es

\section{BIBLIOGRAFÍA}

Alomar, A.I. (1995). L'armament i la defensa a la Mallorca medieval. Terminologia. Palma de Mallorca.

HILT, G. (1876). Waffen-Sammlung Sr. Königlichen Hoheit des Prinzen Carl von Preussen. Mittelalterliche Abteilung. Berlín.

Hinojosa Montalvo, J. (1972). «Coses vedades» en 1404. Valencia.

Hinojosa Montalvo, J. (1975). «Algunos aspectos del comercio valenciano en 1394». En: Homenaje al Dr. D. Juan Reglá Campistol. Valencia, 1975: vol.I, 125-137.

MüLLER, H. (1994). Das Berliner Zeughaus. Vom Arsenal zum Museum. Berlín.

RIQUER, M. (1983). Heráldica Catalana desde l'any 1150 al 1550. Barcelona.

SEvillano Colom, F. (1971). «Mercaderes y navegantes mallorquines (siglos XIII-XV)». En: Mascaró Pasarins, J. (1971). Historia de Mallorca. Palma de Mallorca: 431-520.

Thomas, B. Gamber. O. (1976). Katalog der Leibrüstkamer. I Teil. Der Zeitraum von 500 bis 1530. Wien.

VAlenCia DE DON JuAN, Conde Viudo de [Crooke y Navarrot, J.B.] (1898). Catálogo HistóricoDescriptivo de la Real Armería de Madrid. Madrid. 